

\title{
NOTE ON LINEARITY OF REARRANGEMENT-INVARIANT SPACES
}

\author{
FILIP SOUDSKÝ
}

Communicated by Javier Soria

\begin{abstract}
For some rearrangement-invariant functional $\|\cdot\|_{X}$ having the lattice property, we give a characterization of linearity of the set $\left\{f:\|f\|_{X}<\infty\right\}$. Afterwards we apply this general abstract theorem in the case of Orlicz-Lorentz spaces.
\end{abstract}

\section{INTRODUCTION}

The concept of rearrangement-invariant spaces plays an important role in analysis and its applications. Since the concept was first presented in the 1930s, it has served a number of important applications, such as partial differential equations and Sobolev space theory. It has been intensively studied since the 1950s, starting with the famous pioneering paper [7] in which the so-called classical Lorentz spaces were introduced. It was also shown in that very same article, however, that the functional which governs these spaces is not necessarily a norm in general. In certain cases these "spaces" do not even need to be linear sets. The main reason for this fact is that the operator which associates a measurable function with its nonincreasing rearrangement is not subadditive. Many authors have studied functional properties of these spaces. In 1990, Sawyer in [9] characterized the normability of classical Lorentz spaces. Many papers, with characterizations of linearity (see [4]) quasinormability (see [3]) and normability ([2]) followed (see also [5]).

Copyright 2016 by the Tusi Mathematical Research Group.

Received Apr. 12, 2015; Accepted May 19, 2015.

2010 Mathematics Subject Classification. Primary 46E30; Secondary 46A40.

Keywords. rearrangement-invariant lattice, Lorentz-Orlicz spaces, weighted inequalities, nonincreasing rearrangement, Banach function spaces. 


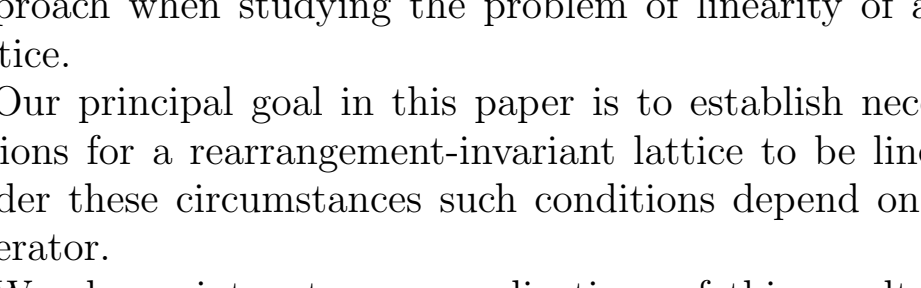

\title{
NOTE ON LINEARITY OF REARRANGEMENT-INVARIANT SPACES
}

\author{
FILIP SOUDSKÝ
}

Communicated by Javier Soria

\begin{abstract}
For some rearrangement-invariant functional $\|\cdot\|_{X}$ having the lattice property, we give a characterization of linearity of the set $\left\{f:\|f\|_{X}<\infty\right\}$. Afterwards we apply this general abstract theorem in the case of Orlicz-Lorentz spaces.
\end{abstract}

\section{INTRODUCTION}

The concept of rearrangement-invariant spaces plays an important role in analysis and its applications. Since the concept was first presented in the 1930s, it has served a number of important applications, such as partial differential equations and Sobolev space theory. It has been intensively studied since the 1950s, starting with the famous pioneering paper [7] in which the so-called classical Lorentz spaces were introduced. It was also shown in that very same article, however, that the functional which governs these spaces is not necessarily a norm in general. In certain cases these "spaces" do not even need to be linear sets. The main reason for this fact is that the operator which associates a measurable function with its nonincreasing rearrangement is not subadditive. Many authors have studied functional properties of these spaces. In 1990, Sawyer in [9] characterized the normability of classical Lorentz spaces. Many papers, with characterizations of linearity (see [4]) quasinormability (see [3]) and normability ([2]) followed (see also [5]).

Copyright 2016 by the Tusi Mathematical Research Group.

Received Apr. 12, 2015; Accepted May 19, 2015.

2010 Mathematics Subject Classification. Primary 46E30; Secondary 46A40.

Keywords. rearrangement-invariant lattice, Lorentz-Orlicz spaces, weighted inequalities, nonincreasing rearrangement, Banach function spaces. 


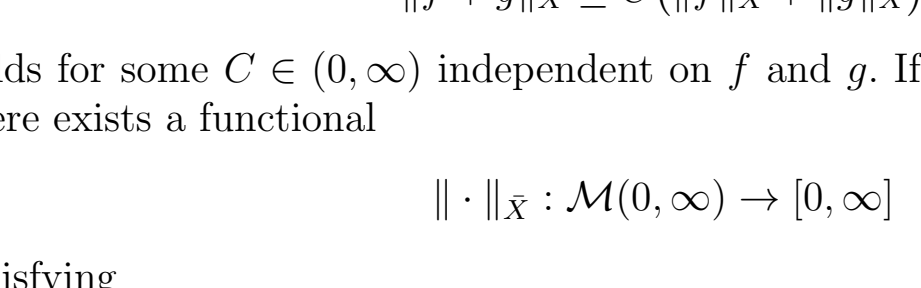

\title{
NOTE ON LINEARITY OF REARRANGEMENT-INVARIANT SPACES
}

\author{
FILIP SOUDSKÝ
}

Communicated by Javier Soria

\begin{abstract}
For some rearrangement-invariant functional $\|\cdot\|_{X}$ having the lattice property, we give a characterization of linearity of the set $\left\{f:\|f\|_{X}<\infty\right\}$. Afterwards we apply this general abstract theorem in the case of Orlicz-Lorentz spaces.
\end{abstract}

\section{INTRODUCTION}

The concept of rearrangement-invariant spaces plays an important role in analysis and its applications. Since the concept was first presented in the 1930s, it has served a number of important applications, such as partial differential equations and Sobolev space theory. It has been intensively studied since the 1950s, starting with the famous pioneering paper [7] in which the so-called classical Lorentz spaces were introduced. It was also shown in that very same article, however, that the functional which governs these spaces is not necessarily a norm in general. In certain cases these "spaces" do not even need to be linear sets. The main reason for this fact is that the operator which associates a measurable function with its nonincreasing rearrangement is not subadditive. Many authors have studied functional properties of these spaces. In 1990, Sawyer in [9] characterized the normability of classical Lorentz spaces. Many papers, with characterizations of linearity (see [4]) quasinormability (see [3]) and normability ([2]) followed (see also [5]).

Copyright 2016 by the Tusi Mathematical Research Group.

Received Apr. 12, 2015; Accepted May 19, 2015.

2010 Mathematics Subject Classification. Primary 46E30; Secondary 46A40.

Keywords. rearrangement-invariant lattice, Lorentz-Orlicz spaces, weighted inequalities, nonincreasing rearrangement, Banach function spaces. 


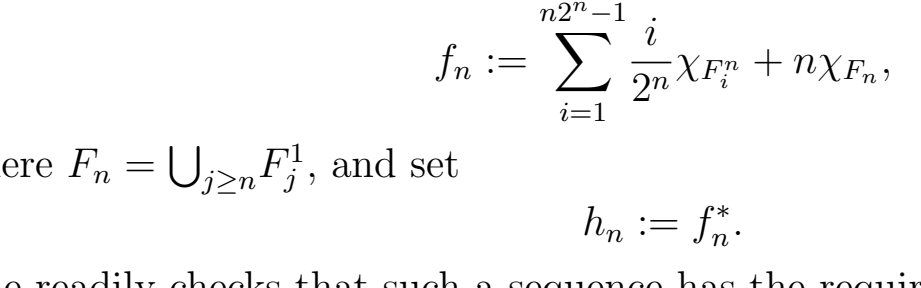

\title{
NOTE ON LINEARITY OF REARRANGEMENT-INVARIANT SPACES
}

\author{
FILIP SOUDSKÝ
}

Communicated by Javier Soria

\begin{abstract}
For some rearrangement-invariant functional $\|\cdot\|_{X}$ having the lattice property, we give a characterization of linearity of the set $\left\{f:\|f\|_{X}<\infty\right\}$. Afterwards we apply this general abstract theorem in the case of Orlicz-Lorentz spaces.
\end{abstract}

\section{INTRODUCTION}

The concept of rearrangement-invariant spaces plays an important role in analysis and its applications. Since the concept was first presented in the 1930s, it has served a number of important applications, such as partial differential equations and Sobolev space theory. It has been intensively studied since the 1950s, starting with the famous pioneering paper [7] in which the so-called classical Lorentz spaces were introduced. It was also shown in that very same article, however, that the functional which governs these spaces is not necessarily a norm in general. In certain cases these "spaces" do not even need to be linear sets. The main reason for this fact is that the operator which associates a measurable function with its nonincreasing rearrangement is not subadditive. Many authors have studied functional properties of these spaces. In 1990, Sawyer in [9] characterized the normability of classical Lorentz spaces. Many papers, with characterizations of linearity (see [4]) quasinormability (see [3]) and normability ([2]) followed (see also [5]).

Copyright 2016 by the Tusi Mathematical Research Group.

Received Apr. 12, 2015; Accepted May 19, 2015.

2010 Mathematics Subject Classification. Primary 46E30; Secondary 46A40.

Keywords. rearrangement-invariant lattice, Lorentz-Orlicz spaces, weighted inequalities, nonincreasing rearrangement, Banach function spaces. 


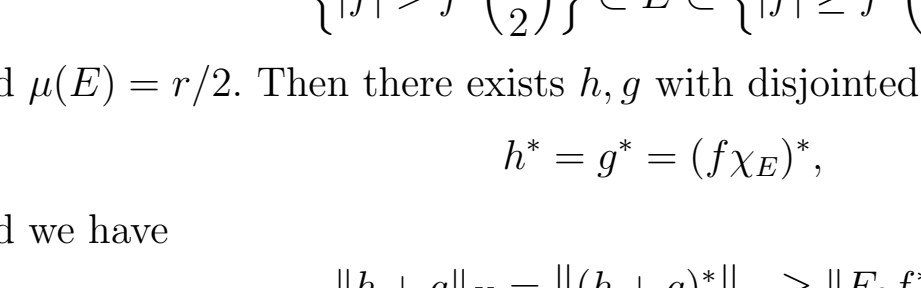

\title{
NOTE ON LINEARITY OF REARRANGEMENT-INVARIANT SPACES
}

\author{
FILIP SOUDSKÝ
}

Communicated by Javier Soria

\begin{abstract}
For some rearrangement-invariant functional $\|\cdot\|_{X}$ having the lattice property, we give a characterization of linearity of the set $\left\{f:\|f\|_{X}<\infty\right\}$. Afterwards we apply this general abstract theorem in the case of Orlicz-Lorentz spaces.
\end{abstract}

\section{INTRODUCTION}

The concept of rearrangement-invariant spaces plays an important role in analysis and its applications. Since the concept was first presented in the 1930s, it has served a number of important applications, such as partial differential equations and Sobolev space theory. It has been intensively studied since the 1950s, starting with the famous pioneering paper [7] in which the so-called classical Lorentz spaces were introduced. It was also shown in that very same article, however, that the functional which governs these spaces is not necessarily a norm in general. In certain cases these "spaces" do not even need to be linear sets. The main reason for this fact is that the operator which associates a measurable function with its nonincreasing rearrangement is not subadditive. Many authors have studied functional properties of these spaces. In 1990, Sawyer in [9] characterized the normability of classical Lorentz spaces. Many papers, with characterizations of linearity (see [4]) quasinormability (see [3]) and normability ([2]) followed (see also [5]).

Copyright 2016 by the Tusi Mathematical Research Group.

Received Apr. 12, 2015; Accepted May 19, 2015.

2010 Mathematics Subject Classification. Primary 46E30; Secondary 46A40.

Keywords. rearrangement-invariant lattice, Lorentz-Orlicz spaces, weighted inequalities, nonincreasing rearrangement, Banach function spaces. 


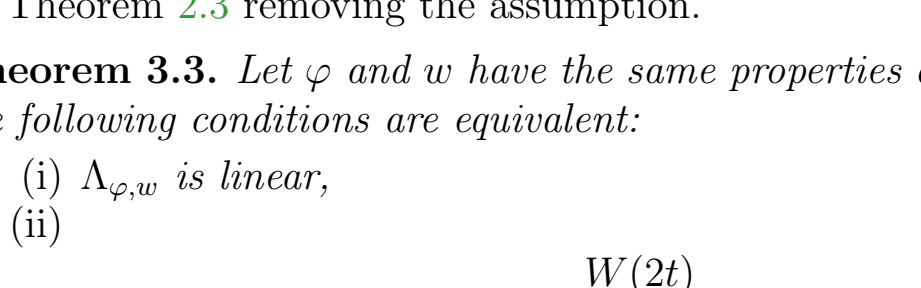

\title{
NOTE ON LINEARITY OF REARRANGEMENT-INVARIANT SPACES
}

\author{
FILIP SOUDSKÝ
}

Communicated by Javier Soria

\begin{abstract}
For some rearrangement-invariant functional $\|\cdot\|_{X}$ having the lattice property, we give a characterization of linearity of the set $\left\{f:\|f\|_{X}<\infty\right\}$. Afterwards we apply this general abstract theorem in the case of Orlicz-Lorentz spaces.
\end{abstract}

\section{INTRODUCTION}

The concept of rearrangement-invariant spaces plays an important role in analysis and its applications. Since the concept was first presented in the 1930s, it has served a number of important applications, such as partial differential equations and Sobolev space theory. It has been intensively studied since the 1950s, starting with the famous pioneering paper [7] in which the so-called classical Lorentz spaces were introduced. It was also shown in that very same article, however, that the functional which governs these spaces is not necessarily a norm in general. In certain cases these "spaces" do not even need to be linear sets. The main reason for this fact is that the operator which associates a measurable function with its nonincreasing rearrangement is not subadditive. Many authors have studied functional properties of these spaces. In 1990, Sawyer in [9] characterized the normability of classical Lorentz spaces. Many papers, with characterizations of linearity (see [4]) quasinormability (see [3]) and normability ([2]) followed (see also [5]).

Copyright 2016 by the Tusi Mathematical Research Group.

Received Apr. 12, 2015; Accepted May 19, 2015.

2010 Mathematics Subject Classification. Primary 46E30; Secondary 46A40.

Keywords. rearrangement-invariant lattice, Lorentz-Orlicz spaces, weighted inequalities, nonincreasing rearrangement, Banach function spaces. 


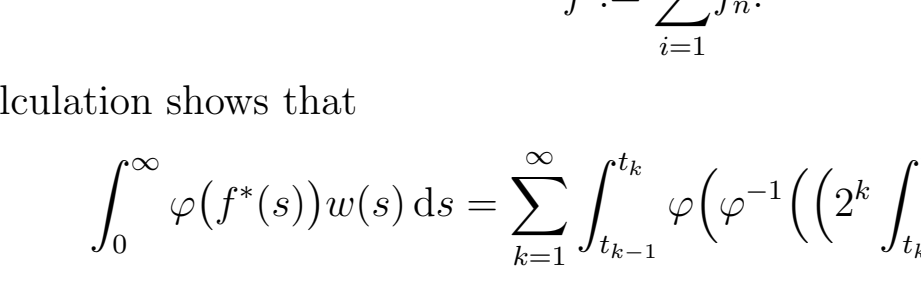

\title{
NOTE ON LINEARITY OF REARRANGEMENT-INVARIANT SPACES
}

\author{
FILIP SOUDSKÝ
}

Communicated by Javier Soria

\begin{abstract}
For some rearrangement-invariant functional $\|\cdot\|_{X}$ having the lattice property, we give a characterization of linearity of the set $\left\{f:\|f\|_{X}<\infty\right\}$. Afterwards we apply this general abstract theorem in the case of Orlicz-Lorentz spaces.
\end{abstract}

\section{INTRODUCTION}

The concept of rearrangement-invariant spaces plays an important role in analysis and its applications. Since the concept was first presented in the 1930s, it has served a number of important applications, such as partial differential equations and Sobolev space theory. It has been intensively studied since the 1950s, starting with the famous pioneering paper [7] in which the so-called classical Lorentz spaces were introduced. It was also shown in that very same article, however, that the functional which governs these spaces is not necessarily a norm in general. In certain cases these "spaces" do not even need to be linear sets. The main reason for this fact is that the operator which associates a measurable function with its nonincreasing rearrangement is not subadditive. Many authors have studied functional properties of these spaces. In 1990, Sawyer in [9] characterized the normability of classical Lorentz spaces. Many papers, with characterizations of linearity (see [4]) quasinormability (see [3]) and normability ([2]) followed (see also [5]).

Copyright 2016 by the Tusi Mathematical Research Group.

Received Apr. 12, 2015; Accepted May 19, 2015.

2010 Mathematics Subject Classification. Primary 46E30; Secondary 46A40.

Keywords. rearrangement-invariant lattice, Lorentz-Orlicz spaces, weighted inequalities, nonincreasing rearrangement, Banach function spaces. 


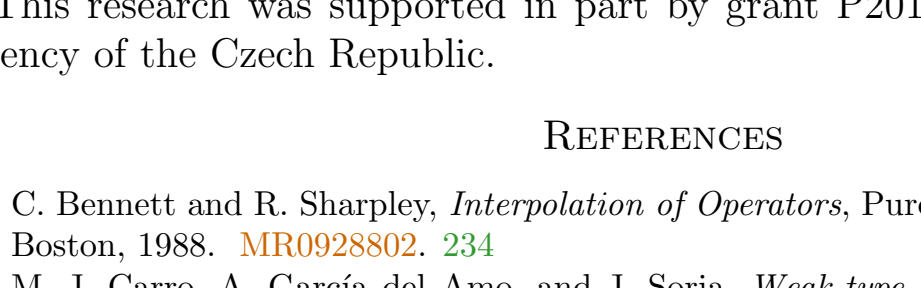

\title{
NOTE ON LINEARITY OF REARRANGEMENT-INVARIANT SPACES
}

\author{
FILIP SOUDSKÝ
}

Communicated by Javier Soria

\begin{abstract}
For some rearrangement-invariant functional $\|\cdot\|_{X}$ having the lattice property, we give a characterization of linearity of the set $\left\{f:\|f\|_{X}<\infty\right\}$. Afterwards we apply this general abstract theorem in the case of Orlicz-Lorentz spaces.
\end{abstract}

\section{INTRODUCTION}

The concept of rearrangement-invariant spaces plays an important role in analysis and its applications. Since the concept was first presented in the 1930s, it has served a number of important applications, such as partial differential equations and Sobolev space theory. It has been intensively studied since the 1950s, starting with the famous pioneering paper [7] in which the so-called classical Lorentz spaces were introduced. It was also shown in that very same article, however, that the functional which governs these spaces is not necessarily a norm in general. In certain cases these "spaces" do not even need to be linear sets. The main reason for this fact is that the operator which associates a measurable function with its nonincreasing rearrangement is not subadditive. Many authors have studied functional properties of these spaces. In 1990, Sawyer in [9] characterized the normability of classical Lorentz spaces. Many papers, with characterizations of linearity (see [4]) quasinormability (see [3]) and normability ([2]) followed (see also [5]).

Copyright 2016 by the Tusi Mathematical Research Group.

Received Apr. 12, 2015; Accepted May 19, 2015.

2010 Mathematics Subject Classification. Primary 46E30; Secondary 46A40.

Keywords. rearrangement-invariant lattice, Lorentz-Orlicz spaces, weighted inequalities, nonincreasing rearrangement, Banach function spaces. 